\title{
论文
}

\section{金属基底层上NiO(111)外延薄膜的变温电阻开关 特性与隧穿机制}

\author{
魏明龙, 江雪, 王颖玥, 赖芮涟, 王建波, 邱晓燕 ${ }^{*}$ \\ 西南大学物理科学与技术学院, 重庆 400715 \\ *联系人, E-mail: qxy2001@swu.edu.cn \\ 收稿日期: 2018-09-18; 接受日期: 2018-11-29; 网络出版日期: 2019-03-06 \\ 国家自然科学基金(编号: 11274257)和中央高校基本科研业务费专项资金(编号: XDJK2018B034, XDJK2019D034)资助项目
}

\begin{abstract}
摘要 利用射频磁控溅射方法在 $\mathrm{Pt}$ 层上制备了沿 $<111>$ 晶向外延生长的 $\mathrm{NiO}$ 薄膜, 研究了其晶体微结构和变温电 阻开关特性。微结构观测分析发现, $\mathrm{NiO}(111)$ 薄膜与 $\mathrm{Pt}$ 层满足“立方-立方”晶格生长关系。在周期性外电场作用下, 薄膜中氧空位的定向漂移和电极附近银离子的氧化还原反应相互促进，使得在较低的翻转电压下即可在薄膜中 形成周期性导通/截断的氧空位导电丝通道，从而使得 $\mathrm{Ag} / \mathrm{NiO}(111) / \mathrm{Pt}$ 存储单元在室温至 $80^{\circ} \mathrm{C}$ 测试温度范围里的 电流-电压曲线均呈现稳定的双极性电阻开关特性。运用Arrhenius作图法以及肖特基热激发隧穿公式拟合薄膜高 阻态电流-温度曲线符合线性关系，表明薄膜高阻态漏电流符合肖特基热激发隧穿机制.
\end{abstract}

关键词 $\mathrm{NiO}(111)$ 外延薄膜, 变温, 电阻开关特性, 隧穿机制

PACS: $73.40 . \mathrm{Rw}, 77.84 . \mathrm{Bw}, 73.61 . \mathrm{Ey}$

现代物联网科技的快速发展, 对存储器读写速度 和存储容量的要求进一步提高, 传统非易失性存储器 已逐渐无法满足要求. 2010年, 基于金属-氧化物-金属 三明治结构的氧化物薄膜电阻开关随机存储器(简称 ReRAM) 在沉寂近 40 年后重新成为存储器研究领域的 热点之一. 短短 8 年时间, ReRAM凭借可媲美Flash存储 器的读/写速度 $(\sim 5 \mathrm{~ns})^{[1]}$ 、高达 $\sim 4 \mathrm{~F}^{2}$ 的存储密度 ${ }^{[2]}$ 、大 于 $10^{9}$ 次的读/写周期 ${ }^{[3]}$ 、超过 10 年的有效存储时间以 及降低至 $\mathrm{pJ}$ 量级的功耗和兼容传统半导体制备工艺技 术等显著优势，成为最具应用潜力的下一代非易失性
存储器件之一.

所谓电阻开关效应，指的是在周期性外电场作用 下，薄膜电阻在高/低电阻态之间发生稳定可逆的跳 变. 从1964年报道发现 $\mathrm{NiO}$ 薄膜具有电阻开关特征 ${ }^{[4]}$ 至今, 研究者们已陆续在多种二元及多元氧化物 ${ }^{[-7]}$ 、 固体电解质 ${ }^{[8]}$ 和有机材料 ${ }^{[9]}$ 等多种材料体系中发现了 类似的电阻开关特征. $\mathrm{NiO}$ 作为最早被研究的一种二 元金属氧化物，原料丰富且易制备，是最有望应用于 下一代商用ReRAM存储器件的材料体系之一. 在长达 半个多世纪的时间里, 研究者们从物性、机理、器件

引用格式: 魏明龙, 江雪, 王颖玥, 等. 金属基底层上 $\mathrm{NiO}(111)$ 外延薄膜的变温电阻开关特性与隧穿机制. 中国科学: 物理学 力学 天文学, 2019, 49: 047302 Wei M L, Jiang X, Wang Y Y, et al. Temperature-dependence of resistive switching behaviors and tunneling mechanism of (111) epitaxial NiO films on metal seed layer (in Chinese). Sci Sin-Phys Mech Astron, 2019, 49: 047302, doi: 10.1360/SSPMA2018-00323 
设计和集成等方面对 $\mathrm{NiO}$ 薄膜的电阻开关特性进行 了广泛深入地研究. 到目前为止, 人们已先后在多晶 $\mathrm{NiO}$ 薄膜的存储单元中观测到了单极性 ${ }^{[10-12]}$ 、双极 性 ${ }^{[13-17]}$ 和阈值型 ${ }^{[18,19]}$ 三种类型的电阻开关特性. 除材 料晶体结构外, 器件制备工艺和电极种类也在很大程 度上影响其电阻开关性能参数. 非晶/多晶 $\mathrm{NiO}$ 薄膜由 于物性的各向同性, 在薄膜内缺陷浓度较高的情况下, 膜内导电细丝通道的形成/断裂普遍具有无序性和不 可控性, 因此器件性能稳定性较差. 相比之下, 沿某一 特定晶向择优生长的外延或单晶 $\mathrm{NiO}$ 薄膜由于不同晶 面具有不同的缺陷态浓度, 从而可以对薄膜缺陷的空 间分布实现一定程度的调控, 其电阻开关器件性能参 数也相对具有更好的稳定性和一致性 ${ }^{[20,21]}$. 我们文献 调研发现, 沿单一晶向外延生长的 $\mathrm{NiO}$ 薄膜通常制备 在氧化物单晶补底上 (如 $\mathrm{MgO}^{[22]}$, 掺 $\mathrm{Nb}$ 的 $\mathrm{SrTiO}_{3}{ }^{[23]}$ 和 $\mathrm{SrRuO}_{3}{ }^{[24]}$ 等), 直接在金属导电层上制备单一晶向 $\mathrm{NiO}$ 薄膜的报道还相对较少. 2017年, 我们成功在Pt金属层 上制备出沿 $<001>$ 晶向外延生长的 $\mathrm{NiO}(001)$ 薄膜 ${ }^{[25]}$, 其不仅具有优于多晶 $\mathrm{NiO}$ 薄膜的电阻开关特性, 而且 翻转电压的弥散性得到大幅改善. 而本文主要报道我 们在 $\mathrm{Pt}$ 金属层上沿 $<111>$ 晶向外延生长的 $\mathrm{NiO}(111)$ 薄 膜的电阻开关特性及其随测试温度的变化, 并利用 $\mathrm{Ar}$ rhenius公式拟合其电流-温度曲线, 探讨器件的漏电流 隧穿机制.

\section{1 实验}

选用 $\mathrm{MgO}(001)$ 单晶基片为祄底, 按照文献[26]所 述的方法进行清洗和预退火处理后, 放入基础真空为 $2 \times 10^{-4} \mathrm{~Pa}$ 的腔体备用. 接下来以纯度为 $99.99 \%$ 的 $\mathrm{Pt}$ 金 属靶为溅射靶源, 在总气压为 $2.7 \mathrm{~Pa}$, 氧分压为 $15 \%$ 的 $\mathrm{Ar}+\mathrm{O}_{2}$ 混合气氛中, $700^{\circ} \mathrm{C}$ 衬底温度下, 以 $25 \mathrm{~W}$ 溅射功 率磁控溅射厚约 $50 \mathrm{~nm}$ 的 $\operatorname{Pt}(001)$ 金属薄膜为基底层兼 下电极. 我们前期研究结果表明, 高温无氧环境中制备 的Pt金属薄膜由于存在高温钝化现象, 从而易导致 $\mathrm{Pt}$ 薄膜表面出现纳米微孔 ${ }^{[27]}$. 在浌射氛围中引入 $15 \%$ 的 氧气, 不仅可以降低高温下 $\mathrm{MgO}$ 单晶祄底表面的氧空 位 ${ }^{[28]}$, 从而更有利于Pt薄膜的外延生长; 而且适量的 氧气可以有效地在高温溅射过程中将Pt靶材中残留的 极少量硫杂质氧化成气态硫化物并析出 $\mathrm{Pt}$ 薄膜表 面 ${ }^{[29]}$, 从而生成更纯净且表面无微孔的 $\mathrm{Pt}$ 金属层. 接下
来，选用纯度为 $99.99 \%$ 的 $\mathrm{Ni}$ 金属靶为溅射靶源，在腔 体里充入总气压为 $0.5 \mathrm{~Pa}$ 的 $\mathrm{Ar}+\mathrm{O}_{2}$ 混合气氛 $\left(\mathrm{Ar}: \mathrm{O}_{2}\right.$ 流量 比为 $1: 4)$, 在 $680^{\circ} \mathrm{C}$ 祄底温度, $60 \mathrm{~W}$ 溅射功率下在 $\mathrm{Pt}$ 金 属层上沉积制备 $\mathrm{NiO}$ 薄膜. 利用Dektak6M型光学台阶 仪标定出固定时间沉积的 $\mathrm{NiO}$ 薄膜厚度, 然后除以沉 积时间即可标定出该制备条件下薄膜平均沉积速率约 为 $0.6 \mathrm{~nm} / \mathrm{min}$. 根据薄膜平均沉积速率, 通过控制沉积 时间制备厚约 $100 \mathrm{~nm}$ 的 $\mathrm{NiO}$ 薄膜样品. 最后, 在 $\mathrm{NiO}$ 薄 膜样品表面覆盖孔径为 $0.2 \mathrm{~mm}$ 的多孔不锈钢掩模板, 在 $0.5 \mathrm{~Pa}$ 氩气氛围中以 $25 \mathrm{~W}$ 功率室温直流溅射厚约 $150 \mathrm{~nm}$ 的 $\mathrm{Ag}$ 电极, 形成 $\mathrm{Ag} / \mathrm{NiO} / \mathrm{Pt}$ 三明治结构单元.

分别利用 JSM-7100F型热场发射扫描电子显微镜 ( SEM) 和 JEM-2 $100 \mathrm{~F}$ 型高分辨透射电子显微镜 (HRTEM)观测薄膜表面形貌和样品横截面微结构; 利 用Bede D1型X射线四圆衍射仪(X-ray Four-circle Diffractometer, XRD)探测分析薄膜物相和晶体结构; 最后 利用Keithley2400高精密源表表征了不同测试温度下 $\mathrm{Ag} / \mathrm{NiO} / \mathrm{Pt}$ 存储单元的电流-电压 $(I-V)$ 曲线.

\section{2 结果与讨论}

\section{$2.1 \mathrm{NiO}$ 薄膜表面形貌和样品微结构观测}

图1(a)展示的是优化条件下沉积制备的Pt金属基 底层表面形貌的SEM照片. 照片清晰地显示出Pt基底 层表面平整致密无“针孔”, 呈现 $<001>$ 晶向外延薄膜 典型的层状台阶表面形貌. 图1(b)展示的是沉积制备 $\mathrm{NiO}$ 薄膜表面的SEM照片, 三角雉形晶粒密排形成致 密的膜面, 统计平均粒径约为 $63 \mathrm{~nm}$. 图1(c)为沉积在 $\mathrm{Pt}$ 基底层上 $\mathrm{NiO}$ 薄膜样品的横截面HRTEM照片, 由照 片清晰可见 $\mathrm{NiO}$ 晶粒沿着 $<111>$ 晶向密排堆积形成“大 牙交错” 的三角雉膜面轮廓曲线, 相邻晶粒之间形成 垂直膜面的“倒Y形” 晶界. 图1(d) 则展示了用对数纵 坐标绘制的 $\mathrm{NiO}$ 薄膜样品的XRD衍射谱, 为便于对比, 图1(d)中还绘制出了未沉积 $\mathrm{NiO}$ 薄膜的 $\mathrm{MgO}(001)$ 祄底 在覆盖Pt基底层前后的XRD衍射谱. 由图1(d)可知, 除 观测到 $\mathrm{MgO}(001)$ 单晶祄底的(002) 晶面衍射峰和若干 祄底杂质峰(星号*标注), 只观测到了来源于 $\mathrm{Pt}$ 基底层 的(002)晶面衍射峰以及属于 $\mathrm{NiO}$ 薄膜的(111)和(222) 两个晶面衍射峰, 这初步表明本文实验沉积制备的 $\mathrm{Pt}$ 基底层沿 $<001>$ 晶向外延生长, 而 $\mathrm{NiO}$ 薄膜则沿 $<111>$ 晶向取向生长. 

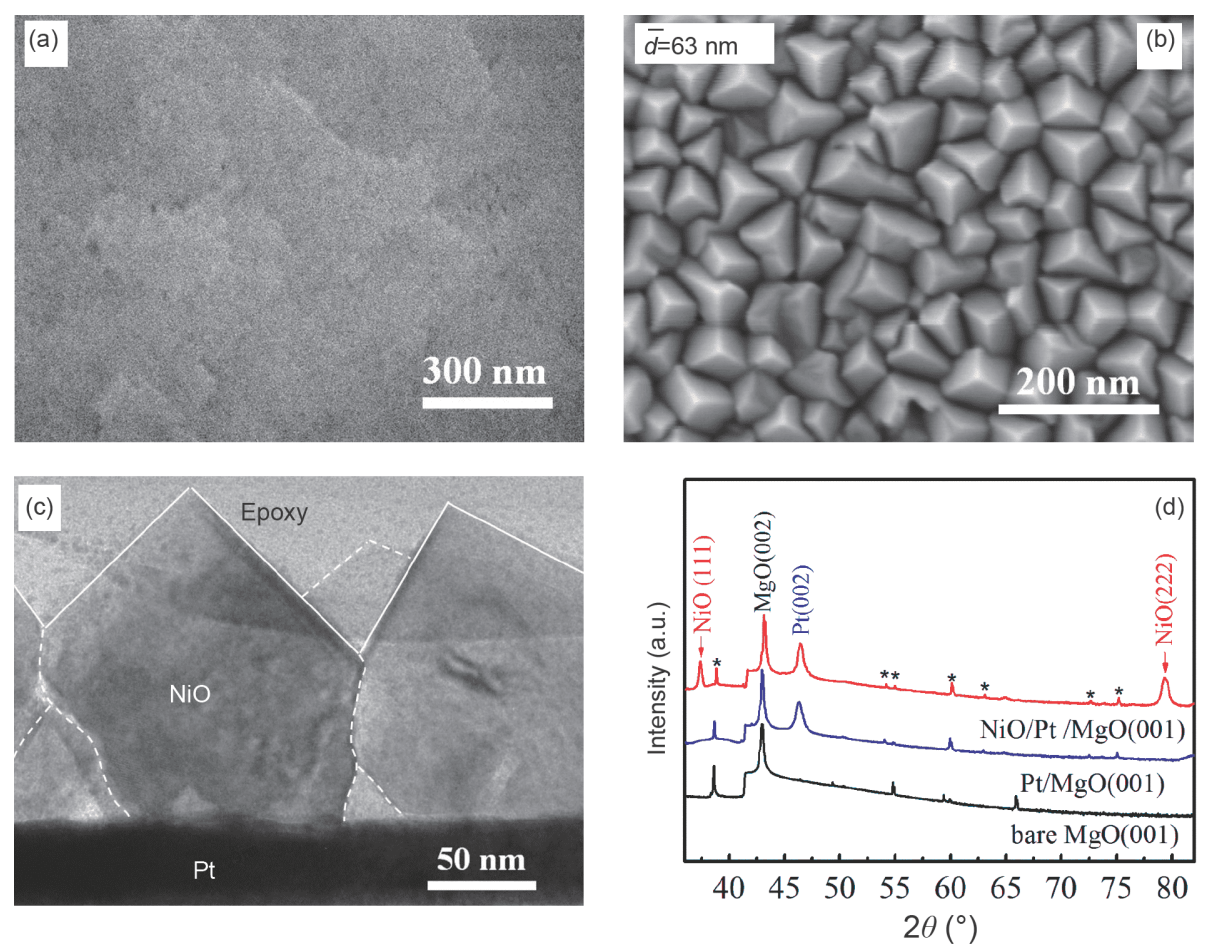

图 1 (网络版彩图)Pt基底层的(a)表面SEM照片, NiO薄膜样品的(b)表面SEM照片, (c)横截面HRTEM照片和(d)XRD图. 为便 于对比, 图1(d)中还绘制出了覆盖Pt基底层前后的 $\mathrm{MgO}(001)$ 衬底的XRD图谱

Figure 1 (Color online) SEM images for the Pt seed layer (a) and the as-deposited NiO film (b). (c) Cross-sectional HRTEM image and (d) XRD spectrum for the $\mathrm{NiO}$ sample deposited on Pt-coated $\mathrm{MgO}(001)$ substrate. XRD spectra for bare $\mathrm{MgO}(001)$ substrates coated with/without Pt seed layer are also shown in Figure 1(d) for reference.

为进一步探测 $\mathrm{MgO} / \mathrm{Pt}$ 和 $\mathrm{Pt} / \mathrm{NiO}$ 界面之间的晶格 取向生长关系, 我们利用 $\mathrm{X}$ 射线面内扫描技术分别在 $\mathrm{MgO}\{111\}, \mathrm{Pt}\{111\}$ 和 $\mathrm{NiO}\{200\}$ 晶面内对样品进行了 $\phi$ 扫描, 所得图谱如图2所示. 对比图2(a)和(b)可知, $\mathrm{MgO}$ $\{111\}$ 和Pt $\{111\}$ 晶面内的 $\phi$ 扫描谱线分别在相同的扫 描角度出现了 4 个间隔为 $90^{\circ}$ 的衍射峰, 这不仅证明 $\mathrm{Pt}$ 基底层具有和 $\mathrm{MgO}(001)$ 单晶祄底相同的四重对称轴, 而且表明二者之间满足 “立方-立方”的晶格外延生长 模式, 即 $\mathrm{Pt}$ 基底层沿 $<001>$ 晶向外延生长在 $\mathrm{MgO}(001)$ 的单晶祄底上. 相比之下, $\mathrm{NiO}\{200\}$ 晶面内的 $\phi$ 扫描图 谱(图2(c)) 则相对复杂, 共计有 12 个衍射峰. 这 12 个衍 射峰可分为 4 组, 每组含 3 个衍射峰, 即有 4 组三重衍射 峰, 图中分别用黑、蓝、绿和紫4种颜色标识不同的 组. 同一组中的 3 个衍射峰的角度间隔均为 $120^{\circ}$, 即具 有三重轴对称性, 这与沿 $<111>$ 晶向取向生长的 $\mathrm{NiO}$ 薄 膜的三重对称轴相吻合. 4组三重衍射峰的出现则表明 $\mathrm{NiO}(111)$ 薄膜中分布着 4 种三重旋转晶畴, 这是因为 $\mathrm{NiO}$ 也属于立方晶系结构. 综上所述, $\mathrm{NiO}$ 薄膜沿着
$<111>$ 晶向取向生长在 $\operatorname{Pt}(001)$ 外延金属基底层上.

\section{$2.2 \mathrm{Ag} / \mathrm{NiO}(111) / \mathrm{Pt}$ 存储单元的电阻开关特性}

我们分别制备了厚度为 50,100 和 $150 \mathrm{~nm}$ 的 $\mathrm{NiO}$ (111)薄膜, 研究了薄膜厚度对 $\mathrm{Ag} / \mathrm{NiO}(111) / \mathrm{Pt}$ 存储单 元室温电流-电压 $(I-V)$ 曲线的影响. 如图3(a)所示: $50 \mathrm{~nm}$ 厚的 $\mathrm{NiO}(111)$ 薄膜 $I-V$ 曲线虽然具有双极性电阻 开关特性, 但是高低电阻比 $<10$, 且耐受性极差, 连续循 环 20 个周期后, 循环电压扫描的 $I-V$ 曲线彼此重合, 不 再具有电阻开关特性. 而 $100 \mathrm{~nm}$ 厚的 $\mathrm{NiO}(111)$ 薄膜 $I-V$ 曲线不仅具有高低电阻比 $>15$ 的双极性电阻开关 特性，而且连续循环 50 个周期后 $I-V$ 曲线没有明显的 变化(图3(b)), 显示出较好的耐受性和稳定性. $\mathrm{NiO}$ (111)薄膜厚度增加到 $150 \mathrm{~nm}$ 后, 薄膜 $I-V$ 曲线高低电阻 比虽然进一步增大, 但是器件跳变电压增大到约 $\pm 1.5 \mathrm{~V}, \mathrm{ON}$ 态漏电流进一步增大, 导致 $I-V$ 曲线稳定性 较差, 连续循环 20 个周期后 $I-V$ 曲线也不再具有阻变特 性, 如图3(c)所示. 综合考虑器件阻变开关参数和器件 


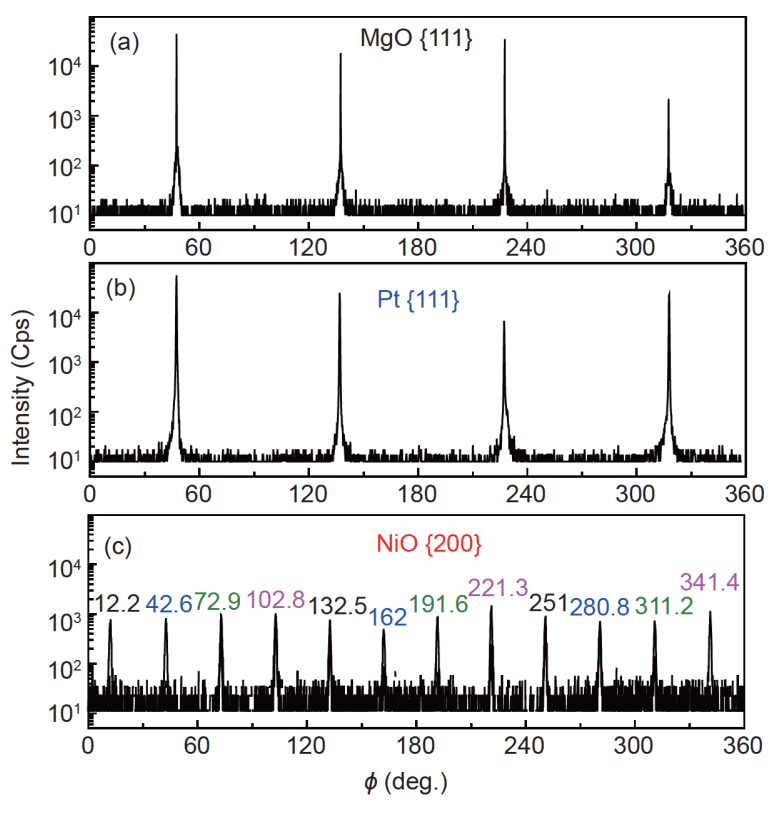

图 2 (网络版彩图) NiO薄膜样品分别在(a) $\mathrm{MgO}\{111\}$, (b) $\mathrm{Pt}\{111\}$ 和(c) $\mathrm{NiO}\{200\}$ 晶面内的X射线 $\phi$ 扫描谱图

Figure 2 (Color online) $\mathrm{X}$-ray $\phi$-scan spectra of $\mathrm{NiO}$ sample taken at (a) $\mathrm{MgO}\{111\}$, (b) $\mathrm{Pt}\{111\}$ and (c) $\mathrm{NiO}\{200\}$ reflections, respectively.

稳定性, 在接下来的研究中, 我们选择厚度为 $100 \mathrm{~nm}$ 的 $\mathrm{NiO}(111)$ 薄膜研究其阻变开关的热稳定性.

接下来, 我们研究 $\mathrm{NiO}(111)$ 薄膜厚度为 $100 \mathrm{~nm}$ 的 $\mathrm{Ag} / \mathrm{NiO}(111) / \mathrm{Pt}$ 存储单元在不同测试温度下的 $I-V$ 曲线. 测试从室温(RT)开始, 每升高 $20^{\circ} \mathrm{C}$ 进行 50 次 $I-V$ 循环扫 描测试. 测试所加栅极偏压扫描步长为 $50 \mathrm{mV}$ ，按照 $0 \rightarrow 1.1 \rightarrow 0 \rightarrow-1.1 \rightarrow 0 \mathrm{~V}$ 的顺序连续扫描 50 个周期，限 制电流为 $0.1 \mathrm{~A}$.

如图4(a)所示, $\mathrm{Ag} / \mathrm{NiO}(111) / \mathrm{Pt}$ 存储单元的室温 $I-V$ 曲线呈现出稳定的双极性电阻开关特性: 零偏压时, 样
品处于高电阻态(HRS); 当正向偏压扫描到约 $0.3 \mathrm{~V}$ 时, 栅电流剧增, 样品从高电阻态HRS跃变到低电阻态 (LRS)，对应存储单元从截止的“OFF”态切换至导通的 “ON”态，完成置位(Set)过程. 在随后的反向电压扫描 过程中, 样品一直处于LRS态, 直至负向偏压增大至约 $-1 \mathrm{~V}$ 时, 栅电流骤减, 样品从LRS恢复到HRS, 存储单 元从“ON”态切换回到“OFF”态，完成复位(Reset)过程， 形成典型的双极性电阻开关回线. 连续循环扫描 50 个 周期的 $I-V$ 曲线呈现出几乎相同的跳变轨迹, 表明其在 室温下具有稳定的双极性电阻开关特性. 图4(b)-(e)分 别展示的是存储单元在 $40,60,80$ 和 $100^{\circ} \mathrm{C}$ 测试温度下 连续测试 50 个扫描周期的 $I-V$ 曲线. 对比可以发现, 随 着测试温度的升高, 存储单元 $I-V$ 曲线依然呈现稳定的 双极性电阻开关特性, 但是回线形状发生明显的变化: 随着测试温度的升高, LRS漏电流没有明显变化, 但 HRS漏电流逐渐增大，从而导致薄膜高低电阻态电阻 比 $\left(R_{\mathrm{H}} / R_{\mathrm{L}}\right)$ 逐渐降低. 当测试温度升高到 $100^{\circ} \mathrm{C}$ 后, 存储 单元HRS和LRS的 $I-V$ 曲线局部相互交迭，不再具有稳 定的电阻开关现象, 如图4(e)所示. 以上对比实验结果 表明, $\mathrm{Ag} / \mathrm{NiO}(111) / \mathrm{Pt}$ 存储单元能保持稳定电阻开关性 能的工作温度区间为 RT $-80^{\circ} \mathrm{C}$.

在实际生产生活应用中除要求器件具有优良的性 能参数外, 还要求器件性质具有稳定的时效性. 因此我 们先后在制备当天和放置第10天表征了同一个器件的 室温 $I-V$ 曲线(见图5(a)和(b)). 然后在放置的第 10 天, 将 该器件升温到 $100^{\circ} \mathrm{C}$ 后再自然冷却至室温, 再次测量 器件的室温 $I-V$ 曲线如图5(c)所示. 对比图5(a)-(c)可发 现, 三次测量的 $I-V$ 曲线除了高阻态电流略有增加外, 其他电阻开关特性参数并没有明显变化, 显示出器件
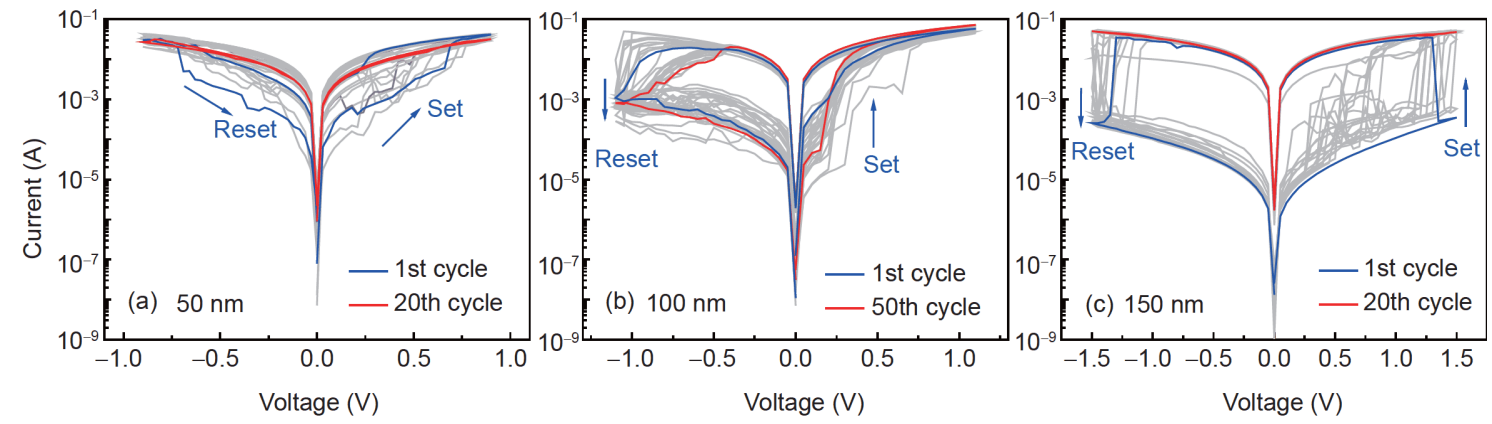

图 3 (网络版彩图)厚度分别为(a) 50, (b) 100 和(c) $150 \mathrm{~nm}$ 的 $\mathrm{NiO}(111$ )薄膜在室温连续循环多个周期的电流-电压曲线 Figure 3 (Color online) Consecutive $I-V$ cycles for (a) 50, (b) 100 and (c) $150 \mathrm{~nm}$-thick $\mathrm{NiO}(111)$ films measured at room temperature (RT), respectively. 

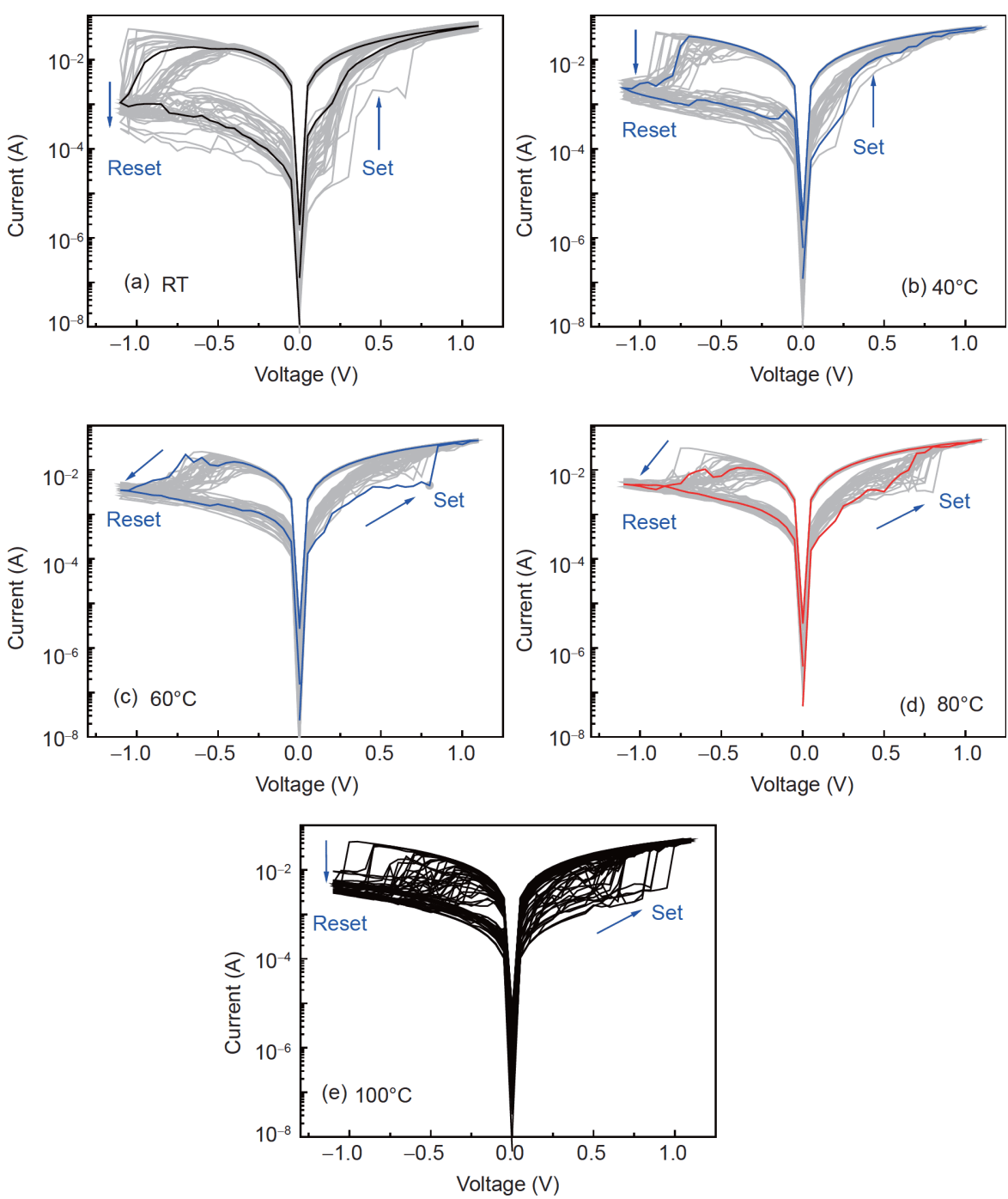

图 4 (网络版彩图)不同测试温度下, $\mathrm{Ag} / \mathrm{NiO}(111) / \mathrm{Pt}$ 存储单元连续循环 50 个周期的电流-电压曲线: (a) RT, (b) 40, (c) 60, (d) 80 和(e) $100^{\circ} \mathrm{C}$

Figure 4 (Color online) 50 consecutive $I-V$ cycles for $\mathrm{Ag} / \mathrm{NiO}(111) / \mathrm{Pt}$ memory cell measured at different temperatures: (a) RT, (b) 40, (c) 60, (d) 80 and (e) $100^{\circ} \mathrm{C}$, respectively.

性能在室温下具有非常稳定的时效性.

接下来, 我们对比研究了室温和 $80^{\circ} \mathrm{C}$ 测试温度下, 存储单元电阻开关主要性能参数的稳定性. 如图6(a) 所示, 当测试温度从室温升高至 $80^{\circ} \mathrm{C}, \mathrm{LRS}$ 电阻 $R_{\mathrm{L}}$ 几 乎不变, 但 $\mathrm{HRS}$ 电阻 $R_{\mathrm{H}}$ 明显减小, 从而导致存储单元 的电阻开关比 $R_{\mathrm{H}} / R_{\mathrm{L}}$ 从 $>15$ 降低至 $5-10$. 虽然 $R_{\mathrm{H}} / R_{\mathrm{L}}$ 随测 试温度的升高有所降低, 但依然满足电阻开关器件的 最低应用要求 $\left(R_{\mathrm{H}} / R_{\mathrm{L}}>5\right)$. 另一方面, 随着测试温度的 升高, 存储单元置位电压 $\left(V_{\mathrm{Set}}\right)$ 从室温时的 $0.25 \mathrm{~V}$ 逐渐 增大到 $0.5 \mathrm{~V}$, 而复位电压 $\left(V_{\text {Reset }}\right)$ 却与之相反, 从室温
时的 $-1 \mathrm{~V}$ 减小到约 $-0.5 \mathrm{~V}$, 如图6(b)所示. 综上所述, 薄膜HRS 电阻 $R_{\mathrm{H}}$ 随测试温度升高显著降低, 同时 $V_{\mathrm{Set}}$ 电压变大而 $V_{\text {Reset }}$ 电压却变小, 存储单元电阻开关效应 明显的温度依赖性意味着薄膜电阻开关机制倾向于热 激发的氧空位缺陷导电细丝通道机制.

由于 $\mathrm{Ag} / \mathrm{NiO}(111) / \mathrm{Pt}$ 存储单元HRS的电流具有明 显的温度依赖性, 因此接下来我们重点研究存储单元 在室温到 $80^{\circ} \mathrm{C}$ 测试范围内 $\mathrm{HRS}$ 时的电流-电压曲线随 测试温度的变化. 如图7(a)所示, 随着测试温度的升高, 相同的栅极偏压下, HRS漏电流逐渐增大. 值得注意的 

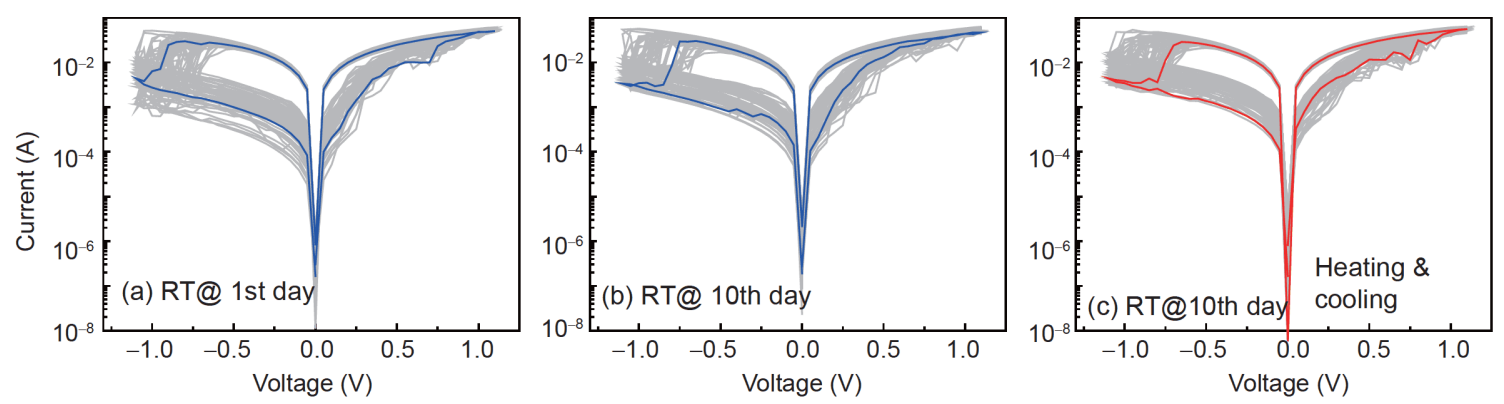

图 5 (网络版彩图) $\mathrm{Ag} / \mathrm{NiO}(111) / \mathrm{Pt}$ 存储单元在室温下分别放置 1 天(a)和 10 天(b), 以及(c)放置 10 天后从室温升温到 $100^{\circ} \mathrm{C}$ 又自 然冷却至室温后连续循环扫描50个周期的电流-电压曲线

Figure 5 (Color online) 50 consecutive $I-V$ cycles for the $\mathrm{Ag} / \mathrm{NiO}(111) / \mathrm{Pt}$ memory cell measured at $\mathrm{RT}$ on (a) the first day, (b) the 10th day and (c) after heating from $\mathrm{RT}$ to $100^{\circ} \mathrm{C}$, then naturally cooling to RT on the 10 th day.
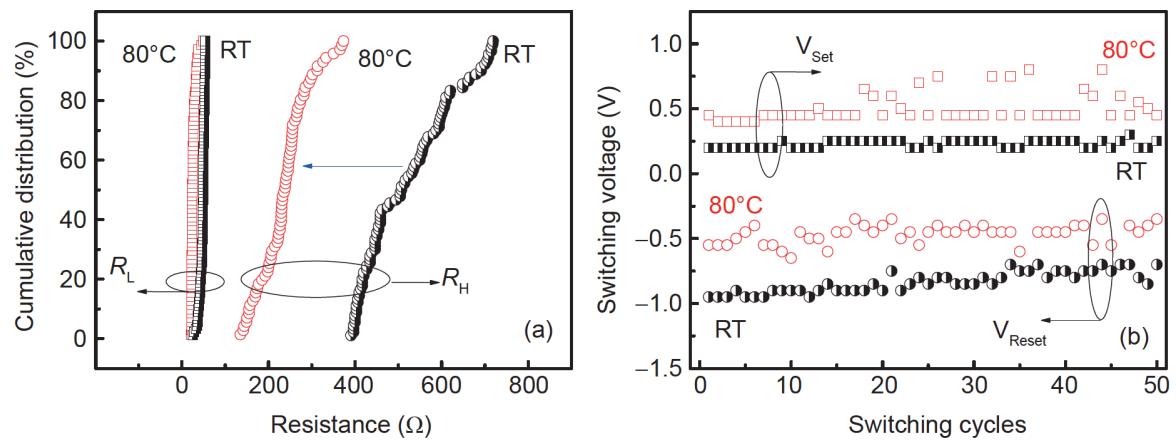

图 6 (网络版彩图) $\mathrm{Ag} / \mathrm{NiO}(111) / \mathrm{Pt}$ 存储单元分别在室温和 $80^{\circ} \mathrm{C}$ 测试温度下的(a)高(低)电阻态阻值 $R_{\mathrm{H}}\left(R_{\mathrm{L}}\right)$ 的累积概率分布图 和(b)置(复)位电压 $V_{\mathrm{Set}}\left(V_{\mathrm{Reset}}\right)$ 随开关周期的变化图

Figure 6 (Color online) (a) Cumulative distribution of $R_{\mathrm{H}}\left(R_{\mathrm{L}}\right)$ and (b) varies of $V_{\mathrm{Set}}\left(V_{\text {Reset }}\right)$ with switching cycles for $\mathrm{Ag} / \mathrm{NiO}(111) / \mathrm{Pt}$ memory cells measured at $\mathrm{RT}$ and $80^{\circ} \mathrm{C}$, respectively.

是，不同于 $\mathrm{NiO}$ 多晶薄膜在HRS态具有的半导体特性 $I-V$ 曲线 ${ }^{[18]}$ ，图7(a)中不同温度下 $\mathrm{NiO}(111)$ 薄膜HRS态 的 $I-V$ 曲线都近似呈线性关系. 这是因为相比非晶/多 晶 $\mathrm{NiO}$ 薄膜内部无序的氧空位缺陷分布, 沿着 $<111>$ 晶 向外延生长的 $\mathrm{NiO}$ 晶粒之间形成垂直上下电极的“倒 $\mathrm{Y}$ 形” 晶界(图1(c)), 使得 $\mathrm{NiO}(111)$ 薄膜中氧离子在较小的 电场作用下，即可沿着垂直晶界漂移形成导通上下电 极的导电丝通道, 从而导致 $\mathrm{NiO}(111)$ 外延薄膜的绝缘 性相对较差, 所以 $I-V$ 曲线近似呈线性关系. 接下来, 我 们利用Arrhenius作图法将图7(a)重新绘制成电流-温度 曲线(图7(b))发现，处于HRS的存储单元在0.5-0.9 V偏 压下的电流与测试温度 $T$ 的Arrhenius 拟合曲线 $(\ln I \propto 1 / T)$ 完全满足线性关系. 根据Arrhenius 公 式 $I \propto A \exp \left[-E_{\mathrm{a}} /\left(k_{\mathrm{B}} T\right)\right]^{[30]}$, 由 Arrhenius拟合曲线的斜 率和玻尔兹曼常数 $k_{\mathrm{B}}$ 计算得到不同偏压下的电子激活 能 $E_{\mathrm{a}}$ 如图7(b)插图所示. 在 $0.5-0.9 \mathrm{~V}$ 的偏压范围内, 薄
膜内电子所需的激活能位于 $0.25-0.30 \mathrm{eV}$ 之间, 远远高 于 $80^{\circ} \mathrm{C}$ 下电子的热动能 $0.03 \mathrm{eV}$, 这表明在 $0.5-0.9 \mathrm{~V}$ 偏 压范围内, 薄膜内热激发的移动电荷数较少, 薄膜依 然可保持较好的绝缘性, 处于高电阻态. 但是加热使 得薄膜与金属电极之间的肖特基势垒降低, 增加了电 子的隧穿机率. 图7(c)展示的是处于HRS时存储单元 分别在0.5-0.9 V偏压下的肖特基热激发隧穿公式拟合 曲线 $\left(\ln \left(I / T^{2}\right) \propto 1 / T\right)$. 不同偏压下的拟合曲线全部满 足线性关系, 这表明加温条件下存储单元HRS漏电流 符合肖特基热激发隧穿机制.

\section{$2.3 \mathrm{Ag} / \mathrm{NiO}(111) / \mathrm{Pt}$ 存储单元双极性电阻开关的物 理机制}

经过研究者们多年实验和理论研究, 大家共识的 金属氧化物薄膜电阻开关物理机制主要有以下两种: 氧空位导电细丝模型 ${ }^{[31]}$ 和金属导电桥模型 ${ }^{[32]}$. 前者适 

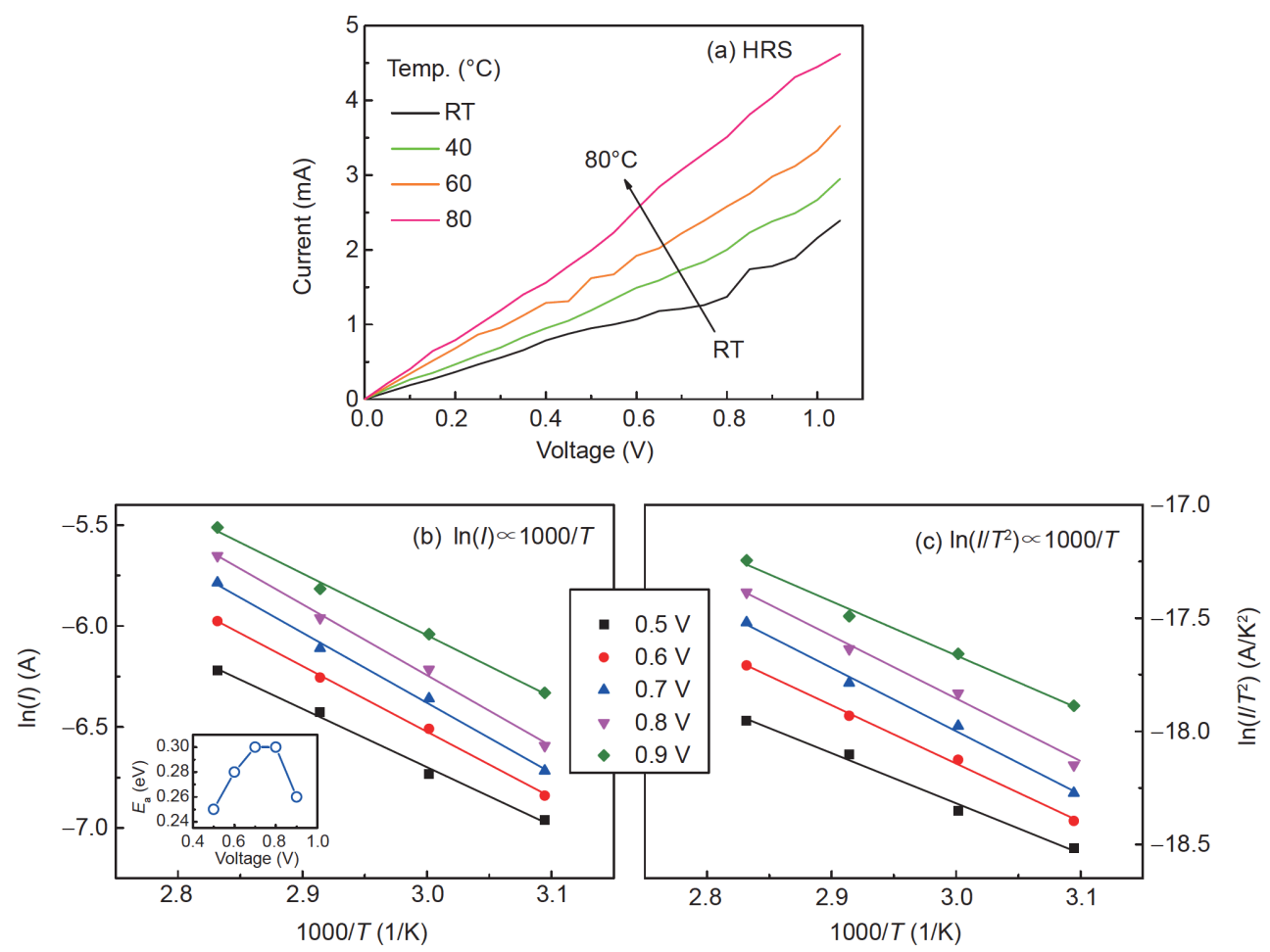

图 7 (网络版彩图) $\mathrm{Ag} / \mathrm{NiO}(111) / \mathrm{Pt}$ 存储单元在 $\mathrm{RT}$ 到 $80^{\circ} \mathrm{C}$ 测试温度范围内的 $(\mathrm{a})$ 高电阻态 $I-V$ 曲线以及在 $0.5-0.9 \mathrm{~V}$ 偏压范围的 (b)Arrhenius拟合曲线和(c)肖特基热激发隧穿公式拟合曲线. 图7(b)插图为根据Arrhenius公式计算出来的不同偏压下的电子激 活能 $E_{\mathrm{a}}$

Figure 7 (Color online) (a) HRS-state $I-V$ curves for $\mathrm{Ag} / \mathrm{NiO}(111) / \mathrm{Pt}$ memory cells measured in the temperature range from $\mathrm{RT}$ to $80^{\circ} \mathrm{C}$. (b) Corresponding Arrhenius form (ln $I$ vs. 1000/T) plots and (c) Schottky tunneling $\left(\ln \left(I / T^{2}\right)\right.$ vs. 1000/T) plots in the bias voltage range from 0.5 to $0.9 \mathrm{~V}$. Inset in (b) shows corresponding electron activation energies $\left(E_{\mathrm{a}}\right)$ extracted from the Arrhenius formula.

用于绝大部分金属氧化物，后者则主要适用于具有较 高电化学反应活性的金属氧化物电解质, 如 $\mathrm{AgO}_{x}$, $\mathrm{AgS}_{x}, \mathrm{AgI}_{x}$ 等. 本文实验研究的 $\mathrm{NiO}$ 薄膜不具有电解质 特性, 但Ni离子具有多重化合价, 而且磁控溅射方法制 备金属氧化物薄膜普遍存在氧配比不足的情况，所以 我们有理由相信本文实验制备 $\mathrm{NiO}$ 薄膜存在一定浓度 的氧空位. 再加上其电阻开关特性具有明显的温度依 赖性，因此我们认为 $\mathrm{NiO}(111)$ 薄膜电阻开关物理机制 也属于氧空位导电细丝模型。值得注意的是, 本文实 验用 $\mathrm{NiO}$ 薄膜层厚约 $100 \mathrm{~nm}$, 却在低于 $\pm 1 \mathrm{~V}$ 的翻转电 压下观测到了双极性电阻开关效应，一方面原因是因 为 $\mathrm{NiO}(111)$ 薄膜中具有较高的氧空位缺陷浓度, 另一 方面是因为本文实验中采用了具有高电化学活性的 $\mathrm{Ag}$ 为电极材料. $\mathrm{Ag}$ 电极中的 $\mathrm{Ag}$ 离子在电场作用下可 与氧离子发生氧化还原反应, 有利地促进了薄膜中氧 空位导电通道的形成和截断, 从而降低了翻转电压. 如图8(a)所示, 在正向电场作用下, 薄膜中的氧离子向
$\mathrm{Ag}$ 电极漂移，同时在薄膜中留下氧空位. 随着到达 $\mathrm{Ag}$ 电极的氧离子越来越多, 电极中的 $\mathrm{Ag}$ 离子与氧离子发 生氧化反应生成 $\mathrm{AgO}_{x}$, 不断消耗陆续漂移到 $\mathrm{Ag}$ 电极的 氧离子. $\mathrm{AgO}_{x}$ 层起到了“氧离子存储池”的作用, 收集 容纳从薄膜中源源不断漂移到 $\mathrm{Ag}$ 电极的氧离子, 从而 导致薄膜中氧空位浓度持续增加, 最终相互连通形成 导电细丝通道, 薄膜从高阻态翻转到低阻态, 完成Set 过程, 如图8(b)所示. 当加上负偏压时, $\mathrm{AgO}_{x}$ 发生还原 反应释放出氧离子, 氧离子在反向电场力的作用下扩 散进入薄膜, 与氧空位复合, 从而导致氧空位导电细 丝通道截断(图8(c)), 薄膜回到高阻态, 完成Reset过程. 随着测试温度的升高, 氧离子无规则热运动加强, 使得 氧空位之间保持稳定相互连通的几率降低, 需要更大 的电场力维持氧离子的定向漂移运动确保导电细丝通 道的稳定导通, 所以随着测试温度的升高, $V_{\mathrm{Set}}$ 电压增 大. 与之相反的是, 氧离子日益加剧的无规则热运动增 大了氧空位导电细丝通道在各处断裂的几率, 从而导 


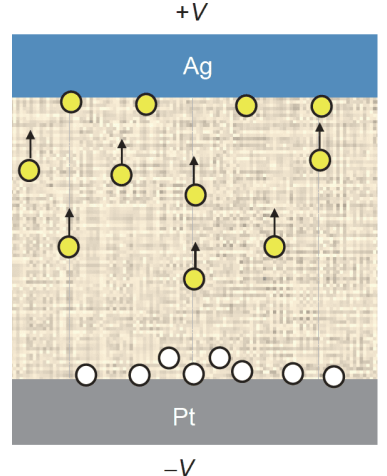

(a) Initial state

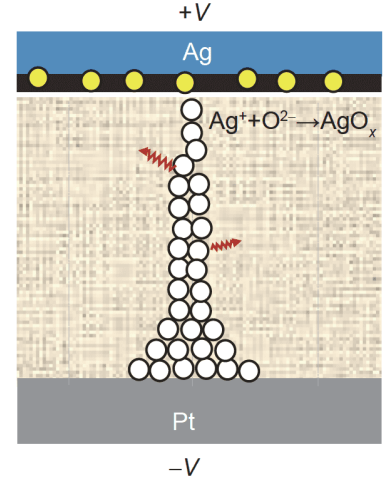

(b) Set process

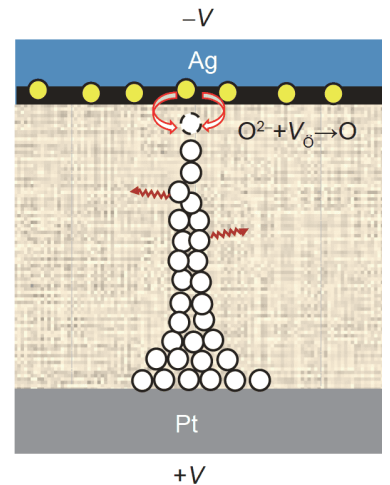

(c) Reset process

$\mathrm{NiO}_{x} \quad \mathrm{~V}_{\ddot{0}} \mathrm{O} \mathrm{O}^{2-}$ ion $\square \mathrm{AgO}_{x}$

图 8 (网络版彩图) $\mathrm{Ag} / \mathrm{NiO}(111) / \mathrm{Pt}$ 存储单元电阻开关物理机制示意图: (a) 在正向电场力作用下, 氧离子向 $\mathrm{Ag}$ 电极定向漂移, 同时在薄膜中留下氧空位; (b) $\mathrm{Ag}$ 离子与氧离子反应生成 $\mathrm{AgO}_{x}$, 不断消耗薄膜中的氧离子, 薄膜中日益增多的氧空位逐渐从 $\mathrm{Pt}$ 电极向 $\mathrm{Ag}$ 电极累积, 最终形成氧空位导电细丝通道, 薄膜完成Set过程, 翻转到低阻态; (c) 当加上负向电场力时, $\mathrm{AgO}_{x}$ 层中的氧 离子被还原释放进入薄膜, 与电极附近的氧空位复合, 导致氧空位导电细丝通道断裂, 薄膜完成Reset过程, 回到高阻态

Figure 8 (Color online) Schematic illustration for formation/rupture of conductive filaments in $\mathrm{Ag} / \mathrm{NiO}(111) / \mathrm{Pt}$ memory cell: (a) when applying a positive electric field oxygen ions drift toward $\mathrm{Ag}$ electrodes, and generate oxygen vacancies in $\mathrm{NiO}(111)$ films. (b) Ag ions react with oxygen ions to form $\mathrm{AgO}_{x}$. This reaction continuously stores oxygen ions in $\mathrm{AgO}_{x}$ layer, and makes the oxygen-vacancy filaments in $\mathrm{NiO}$ film bridge upper and lower electrodes, and finally switch the cell from the OFF-state to the $\mathrm{ON}$-state. (c) When applying a negative electric field, oxygen ions diffuse from $\mathrm{AgO}_{x}$ into the $\mathrm{NiO}(111)$ film, and recombine with oxygen vacancies near upper electrodes. This Reset process results in the rupture of oxygen-vacancy filaments, and makes the cell return to the OFF-state.

致所需的 $V_{\text {Reset }}$ 电压降低.

\section{3 结论}

本文利用射频磁控溅射方法在Pt金属基底层制备 出沿 $<111>$ 晶向生长的 $\mathrm{NiO}$ 薄膜. 晶体微结构观测表明 $\mathrm{NiO}(111)$ 薄膜由三角锥形晶粒密排形成平整致密的膜 面, $\mathrm{NiO} / \mathrm{Pt}$ 界面满足“立方-立方”晶格生长关系. 电学测 试结果表明, 在室温至 $80^{\circ} \mathrm{C}$ 测试温度范围内, $\mathrm{Ag} / \mathrm{NiO}$ (111)/Pt 存储单元均可保持稳定的双极性电阻开关特 性. 运用Arrhenius作图法以及肖特基热激发隧穿机制 拟合高阻态电流-温度曲线满足线性关系, 表明存储单 元高阻态漏电流符合肖特基热激发隧穿机制. 周期性 电场力作用下，薄膜中定向漂移的氧离子和银电极中 $\mathrm{Ag}$ 离子的氧化还原反应相互促进, 使得存储单元在低
于 $\pm 1 \mathrm{~V}$ 的翻转电压下即可形成周期性导通/截断的氧 空位导电丝通道, 从而导致 $\mathrm{Ag} / \mathrm{NiO}(111) / \mathrm{Pt}$ 存储单元呈 现出稳定的双极性电阻开关特性.

需要指出的是, 本文研究的 $\mathrm{NiO}(111)$ 薄膜虽然只 能在不超过 $80^{\circ} \mathrm{C}$ 的温度下保持稳定的电阻开关特性, 但在有降温设备的情况下依然可应用于普通电脑芯片 的存储单元. 如果想让器件在更高的温度下依然保持较 大的 $R_{\mathrm{H}} / R_{\mathrm{L}}$ 比值, 则需要减小器件 $\mathrm{OFF}$ 态时的漏电流. 在 $\mathrm{NiO}$ 外延薄膜的上下表面插入一层绝缘氧化物层是一 种行之有效的解决办法. 我们早期的研究工作 ${ }^{[25]}$ 已证 明插入绝缘 $\mathrm{HfO}_{2}$ 层能将 $\mathrm{NiO}(001)$ 外延薄膜存储单元的 $R_{\mathrm{H}} / R_{\mathrm{L}}$ 比值提高 2 个数量级, 而插入绝缘氧化物界面层的 $\mathrm{NiO}(111)$ 外延薄膜的变温电阻开关特性我们将另文详 述. 另一方面, 本文 $\mathrm{NiO}(111)$ 薄膜电阻开关特性对温度 的敏感性也使得其具有了温控开关器件的应用可能.

\section{参考文献}

1 Lee H Y, Chen P S, Wu T Y, et al. Low power and high speed bipolar switching with a thin reactive Ti buffer layer in robust $\mathrm{HfO}_{2}$ based RRAM. In: Proceedings of IEEE International Electron Devices Meeting (IEDM). San Francisco, 2008. 1-4 
2 Ielmini D. Resistive switching memories based on metal oxides: Mechanisms, reliability and scaling. Semicond Sci Technol, 2016, 31: 063002

3 Yuan F Y, Deng N, Shih C C, et al. Conduction mechanism and improved endurance in $\mathrm{HfO}_{2}$-based RRAM with nitridation treatment. Nanosc Res Lett, 2017, 12: 574

4 Gibbons J F, Beadle W E. Switching properties of thin NiO films. Solid-State Electron, 1964, 7: 785-790

5 Xie W, Hu W, Zou L, et al. Tuning bipolar resistive switching by forming defect dipoles in A-site-deficient perovskite calcium titanate thin films. Appl Phys Express, 2015, 8: 045504

6 Song J, Zhang Y, Xu C, et al. Polar charges induced electric hysteresis of ZnO nano/microwire for fast data storage. Nano Lett, 2011, 11: 28292834

7 Kwon D H, Kim K M, Jang J H, et al. Atomic structure of conducting nanofilaments in $\mathrm{TiO}_{2}$ resistive switching memory. Nat Nanotech, 2010, 5: 148-153

8 Wang Z, Griffin P B, McVittie J, et al. Resistive switching mechanism in $\mathrm{Zn}_{x} \mathrm{Cd}_{1-x} \mathrm{~S}$ nonvolatile memory devices. IEEE Electron Device Lett, 2007, 28: 14-16

9 Wolf C, Nau S, Sax S, et al. Resistive switching based on filaments in metal/PMMA/metal thin film devices. Jpn J Appl Phys, 2015, 54: 120301

10 Liu L, Hou Y, Chen B, et al. Improved unipolar resistive switching characteristics of mixed-NiO $\mathrm{NiO}_{y}$-film-based resistive switching memory devices. Jpn J Appl Phys, 2015, 54: 094201

11 Phark S H, Chae S C. Initial defect configuration in NiO film for reliable unipolar resistance switching of Pt/NiO/Pt structure. J Phys D-Appl Phys, 2015, 48: 155102

$12 \mathrm{Ma} \mathrm{G}$, Tang X, Su H, et al. Effects of electrode materials on bipolar and unipolar switching in NiO resistive switching device. Microelectron Eng, 2014, 129: 17-20

13 Wei L, Li G Q, Zhang W F. Light-induced new memory states in electronic resistive switching of NiO/NSTO junction. J Phys D-Appl Phys, 2016, 49: 045101

$14 \mathrm{Lu} \mathrm{W}$, Xiao J, Wong L M, et al. Probing the ionic and electrochemical phenomena during resistive switching of NiO thin films. ACS Appl Mater Interfaces, 2018, 10: 8092-8101

15 Lee N, Jo W, Liu C, et al. Size dependent bipolar resistance switching of NiO nanodots for low-power and multi-state operation. Nanotechnology, 2014, 25: 415302

16 Liu Z J, Zhang S Y, Huo J Q, et al. Effect of $\mathrm{HfO}_{x}$ buffer layer on the resistive switching characteristics of $\gamma-\mathrm{Fe}_{2} \mathrm{O}_{3}$ nano-particle films (in Chinese). Sci Sin-Phys Mech Astron, 2014, 44: 417-424 [刘志江, 张守英, 霍进迁, 等. $\mathrm{HfO}_{x}$ 缓冲层对 $\gamma-\mathrm{Fe}_{2} \mathrm{O}_{3}$ 纳米微粒膜电阻开关特性的影 响. 中国科学: 物理学 力学 天文学, 2014, 44: 417-424]

17 Huo J Q, Zhang Y Y, Zhu H X, et al. Reversed bipolar resistive switching behaviors modulated by scanning voltage amplitude in polycrystalline $\mathrm{NiO}_{x}$ films (in Chinese). Sci Sin-Phys Mech Astron, 2015, 45: 057701 [霍进迁, 张神扬, 朱华星, 等. 扫描电压幅值调控循环方向的NiOx薄膜 电阻开关特性. 中国科学: 物理学 力学 天文学, 2015, 45: 057701]

18 Wang R X, Zhang T, Li L T, et al. Temperature-dependence of resistive switching behaviors and tunneling mechanism of polycrystalline $\mathrm{NiO}_{x}$ films (in Chinese). Chin Sci Bull, 2017, 62: 1163-1171 [王瑞雪, 张婷, 李鲁涛, 等. NiOx多晶薄膜的变温电阻开关特性与隧穿机制. 科学通 报, 2017, 62: 1163-1171]

19 Guo M Q, Chen Y C, Lin C Y, et al. Unidirectional threshold resistive switching in $\mathrm{Au} / \mathrm{NiO} / \mathrm{Nb}: \mathrm{SrTiO}_{3}$ devices. Appl Phys Lett, 2017, 110: 233504

20 Sullaphen J, Bogle K, Cheng X, et al. Interface mediated resistive switching in epitaxial NiO nanostructures. Appl Phys Lett, 2012, 100: 203115

21 Lee S R, Char K, Kim D C, et al. Resistive memory switching in epitaxially grown NiO. Appl Phys Lett, 2007, $91: 202115$

22 He L, Liao Z M, Wu H C, et al. Memory and threshold resistance switching in Ni/NiO core-shell nanowires. Nano Lett, 2011, 11: 4601-4606

23 Zhu Y, Li M. Bipolar resistive switching characteristic of epitaxial NiO thin film on Nb-doped substrate. Adv Condensed Matter Phys, 2012, 2012: $1-8$

24 Lee S R, Kim H M, Bak J H, et al. Investigation of interface formed between top electrodes and epitaxial NiO films for bipolar resistance switching. Jpn J Appl Phys, 2010, 49: 031102

25 Qiu X Y, Wang R X, Zhang Z, et al. Ultra-low voltage resistive switching of $\mathrm{HfO}_{2}$ buffered (001) epitaxial NiO films deposited on metal seed layers. Appl Phys Lett, 2017, 111: 142103

26 Qiu X Y, Wang R X, Li G Q, et al. Oxygen-dependent epitaxial growth of Pt(001) thin films on MgO(001) by magnetron sputtering. Appl Surf 
Sci, 2017, 406: 212-217

27 Zhang Y Y, Zhu H X, Ji H, et al. Microstructures of epitaxial Pt films on $\mathrm{MgO}$ and $\alpha-\mathrm{Al}_{2} \mathrm{O}_{3}$ single-crystal substrates deposited by magnetron puttering (in Chinese). Chin Sci Bull, 2016, 61: 1008-1015 [张神杨, 朱华星, 姬洪, 等. $\mathrm{MgO} / \alpha-\mathrm{Al}_{2} \mathrm{O}_{3}$ 单晶祄底上磁控浌射 Pt外延薄膜微结构 观测分析. 科学通报, 2016, 61: 1008-1015]

28 Wrighton M S, Ellis A B, Wolczanski P T, et al. Strontium titanate photoelectrodes. Efficient photoassisted electrolysis of water at zero applied potential. J Am Chem Soc, 1976, 98: 2774-2779

29 Ahmad M, Tsong T T. Compositional variations in the near surface layers, an atom-probe study of cosegregation of sulfur in Pt-Rh and Pt-Ir alloys. J Chem Phys, 1985, 83: 388-396

30 Yoon J H, Song S J, Yoo I H, et al. Highly uniform, electroforming-free, and self-rectifying resistive memory in the $\mathrm{Pt} / \mathrm{Ta}_{2} \mathrm{O}_{5} / \mathrm{HfO}{ }_{2-x} / \mathrm{TiN}$ structure. Adv Funct Mater, 2014, 24: 5086-5095

31 Yildirim H, Pachter R. Mechanistic analysis of oxygen vacancy-driven conductive filament formation in resistive random access memory metal/ NiO/metal structures. ACS Appl Mater Interfaces, 2018, 10: 9802-9816

32 Kozicki M N, Barnaby H J. Conductive bridging random access memory-Materials, devices and applications. Semicond Sci Technol, 2016, 31: 113001 


\title{
Temperature-dependence of resistive switching behaviors and tunneling mechanism of (111) epitaxial $\mathrm{NiO}$ films on metal seed layer
}

\author{
WEI MingLong, JIANG Xue, WANG YingYue, LAI RuiLian, \\ WANG JianBo \& QIU XiaoYan* \\ School of Physical Science and Technology, Southwest University, Chongqing 400715, China
}

Since 2000, resistive switching random access memory (ReRAM) based on binary metal oxide has become one of potential candidates for next generation of nonvolatile memory due to its simple structure, high writing/read speed $(\sim 5 \mathrm{~ns})$, good endurance $\left(\sim 10^{9}\right.$ cycles), long retention time ( $\sim 10$ years) and low energy consumption $(\sim$ dozens of pJ). $\mathrm{NiO}$ has been extensively studied as one of promising materials applied for commercial ReRAMs. However, it exhibits diverse resistive switching (RS) behaviors that heavily dependent on fabrication technologies, methods and conditions. Up to now, RS performances and mechanisms of polycrystalline $\mathrm{NiO}$ films have been extensively investigated, while there are few discussions on RS performances of epitaxial NiO films, especially on metal seed layer. On the other hand, with the expanding scope of human activities, researches on stabilities of ReRAM devices under extreme environmental conditions are necessary. In this paper, temperature-dependence of RS behaviors and tunneling mechanism of epitaxial $\mathrm{NiO}(111)$ films on Pt seed layers have been discussed. A set of highly textured $\mathrm{NiO}(111)$ films on Pt seed layers were prepared by means of Radio Frequency magnetron sputtering. Their microstructural characteristics and temperaturedependence of RS performance were investigated. Microstructural investigation demonstrated the "cube-to-cube" lattice relationship of $\mathrm{NiO}(111) / \mathrm{Pt}(001)$ interfaces. Under the applied electric field, the drift of oxygen ions in $\mathrm{NiO}$ film and the redox of Ag ions near upper electrodes result in the formation/rupture of oxygen-vacancy conductive filaments at a set/ reset voltage lower than $\pm 1 \mathrm{~V}$. Moreover, current-voltage $(I-V)$ curves of $\mathrm{Ag} / \mathrm{NiO}(111) / \mathrm{Pt}$ memory cells exhibit stable bipolar switching behaviors in the temperature range from RT to $80^{\circ} \mathrm{C}$. Moreover, their Arrhenius form $(\ln I$ vs. $1000 / T)$ and Schottky tunneling $\left(\ln \left(I / T^{2}\right)\right.$ vs. $\left.1000 / T\right)$ plots are the best linear fitting relationship. It reveals that the leakage current of $\mathrm{Ag} / \mathrm{NiO}(111) / \mathrm{Pt}$ memory cell on high resistance state can be explained by the Schottky tunneling mechanism.

(111) epitaxial NiO film, temperature dependence, resistive switching characteristics, tunneling mechanism

PACS: $73.40 . \mathrm{Rw}, 77.84 . \mathrm{Bw}, 73.61 . \mathrm{Ey}$

doi: $10.1360 /$ SSPMA2018-00323 